\title{
End-User Development in the Internet of Things Era
}

\section{Daniel Tetteroo}

Department of Industrial Design

Eindhoven University of

Technology, the Netherlands

d.tetteroo@tue.n

\section{Panos Markopoulos}

Department of Industrial Design,

Eindhoven University of

Technology, the Netherlands

p.markopoulos@tue.nl

\section{Stefano Valtolina}

Dipartimento di Informatica,

Università degli Studi di Milano,

Italy

valtolin@di.unimi.it

Permission to make digital or hard copies of part or all of this work for personal or classroom use is granted without fee provided that copies are not made or distributed for profit or commercial advantage and that copies bear this notice and the full citation on the first page. Copyrights for third-party components of this work must be honored. For all other uses, contact the Owner/Author.

Copyright is held by the owner/author(s).

CHI'15 Extended Abstracts, Apr 18-23 2015, Seoul, Republic of Korea ACM 978-1-4503-3146-3/15/04.

http://dx.doi.org/10.1145/2702613.2702643
Fabio Paternò

fabio paterno@isti.cnr.it

\section{Volkmar Pipek}

Germany

\section{Margaret Burnett}

Electrical Engineering

Computer Science,

Oregon State University, USA

burnett@eecs.oregonstate.edu

\begin{abstract}
The paradigm of end-user development enables ordinary end-users of computer systems to engage in the modification, extension and even creation of software artifacts. Technology, organization and context are all important aspects that influence endusers' decisions to engage in end-user development. With this workshop, we invite researchers and practitioners to discuss and exchange their experiences on the role of these aspects for end-user development across various domains.
\end{abstract}

\section{Author Keywords}

End-user development; personalization; internet of things; ambient intelligence

\section{ACM Classification Keywords}

H.5.m. Information interfaces and presentation (e.g., $\mathrm{HCI}$ ): Miscellaneous.

\section{Introduction}

A growing trend in $\mathrm{HCI}$ is the adaptation and personalization of interactive applications and devices. Coming from the ultimately impersonal mainframe systems of the 60 's, interactive devices have developed into ambient intelligent computing solutions that are deeply integrated in users' lives and the physical world surrounding us. This trend is expected to continue 
further into the era Internet of Things (IoT) [1] and Quantified Self, where immense amounts of data can be generated by sensor and communication

infrastructures that grow in orders of magnitude, and where applications need to address deeply contextualized user needs. However, this trend of personalization puts ever more pressure on developers and designers of interactive systems. No longer do users accept one-size-fits-all solutions, instead they demand and expect personalized interaction. Despite the sophistication and prevalence of user centered design methods, it is increasingly problematic for developers to anticipate the needs of users at design time. Consequently, it has been proposed that endusers themselves should be enabled create and adapt systems to their liking, in order to satisfy their personal needs [6].

This approach brings about a transformation to the role of the user, who assumes an increasing number of responsibilities traditionally intended for developers, and may need to take on tasks typically carried out by professional programmers. The technology and practices that can support this transformation have traditionally occupied the field of end-user development (EUD). EUD can take different forms, ranging from simply adjusting parameter values, to recording and packaging repetitive interactions in macros, to creating completely new content and system behaviors using scripts, models like spreadsheets, or even full blown programming languages.

In this context, IoT offers unprecedented opportunities to achieve deeper, more meaningful and faster insights by putting the user at the center of informative systems, ambient and personal sensors, communicative tools, and mobile and ubiquitous computing devices [4]. This information feeds a collection of phenomena and facts that characterize the environment in which the user lives and works, and that needs suitable strategies for analyzing them and returning them to the user in in terms of knowledge and ambient intelligence.

Designing, programming or managing this network of things, services, and persons requires metaphors, interfaces, and interaction strategies for supporting users in customization and personalization of the context-dependent application behavior. Therefore, this workshop aims to gather experiences and stimulate discussion on the following topics:

EUD methodologies and end-user empowerment methods: new ideas based on modified user-centered design and participatory design approaches that empower end-users to engage in the modification, extension and creation of interactive systems. These methodologies can bridge the gap between design-time and use-time [7], and anticipate on changing end-user requirements beyond the original design phase.

Tools, services and architectures for supporting EUD: We need new ideas on tools, services and architectural infrastructures able to support EUD in the context of the IoT. Design strategies need to seek a proper balance according to a set of dimensions in order to understand: (1) Volume: the amount of data that will be collected; (2) Variety: the different types and sources of data and interaction strategies that will be integrated and combined; (3) Velocity: the speed at which data will be created, processed and analyzed; (4) Veracity: the level of reliability associated with specific types of actions and or data. 
To apply EUD approaches in this field, strategies and tools need to be developed to support non-expert users in choosing architectural solutions appropriate for their contexts of use and requirements. Therefore, we invite people involved in projects or research activities that aim at allowing the non-experts to modify, extend, or create DIY software supported by suitable architectural choices enabling them to craft their own apps with limited technical skills required. During the workshop, we provide them with the possibility to demonstrate their work, such that other participants can provide feedback hands-on and learn from their experiences.

Contextual factors influencing EUD: Recently it has been recognized that EUD involves much more than providing an end-user with the right tools (e.g., [9]). Rather than a purely technical endeavor, EUD has its place in a socio-technical environment where issues such as learning [8], motivation [2], and organization are important determinants of its success. Especially in the case of ambient intelligent systems, which are by definition bound to a specific context, these issues play an important role. Taking the participants' experiences in deploying and studying EUD systems in various contexts, this workshop aims to generate a better understanding of what contextual factors influence the EUD process.

EUD and social computing: Extending EUD with components of social computing offers many benefits. For example, it gives room to study how end-users developers learn from their peers and how exchanging the content of their creations influences the development process. Exchanging personal creations with peers also promises mutual benefits such as: avoiding double work, collaborative development of solutions that address a shared need, inspiration from others' creations, etc.[5]. By bringing together a varying group of participants from both research and practice, we aim to fuel EUD research with experiences, knowledge, and strategies from social computing.

New evaluation methods for assessing EUD: When designing evaluation tools and selecting evaluation methods, it is useful to consider the working contexts of the users in which apps and services operate. As such, we need to understand how to evaluate the EUD aspects in context of design and development of ambient intelligence solutions. Participants will be encouraged to share their experiences in evaluating EUD environments, in order to arrive at new insights.

\section{Structure of the workshop}

Before the workshop: Potential participants submit a four-page position paper, directly or indirectly addressing the topic and goals of this workshop.

Everyone is asked to include a description of their work and its relation to the workshop theme. Participants are also invited to bring along a demonstrable version of EUD applications they have created, for use in the workshop. This will enrich the collection of EUD applications that the organizers will prepare for experiencing and discussing hands on during the workshop.

During the workshop: The workshop will start off with short, 2-minute presentations by each participant addressing discussion points for the workshop. Then, all participants engage in a 'speed-date' session, during which pairs of participants can informally get to know each other and briefly discuss the points raised during the presentations. After the speed-dates, participants 
receive hands-on experience of the EUD environments that have been brought to the workshop by participants and organizers. Discussants will be exposed to several EUD applications; again the format will be timed discussions around a demonstration where groups of participants rotate around the interactive demonstrations.

After lunch, participants will reflect on their experiences with the EUD environments, to elicit discussion on their success or failure, and the factors that contributed to this. This discussion will be held in sub-groups, each addressing some discussion topics that the organizers will select based on the discussions of the morning session. Discussion tables will present their thoughts to a plenary closing. The overall aim of this discussion is to identify the grand challenges that the field of EUD should pursue in the next 10 years. With this workshop, we aim to abstract away from point solutions characterizing different application domains and technologies, and to avoid an over-siloed future for the field of EUD [3].

After the workshop: All workshop contributions will be collected in dedicated workshop proceedings, published as a technical report on the workshop's webpage. Provided that the quality of submissions allows it, we plan to set up a special issue on the workshop topic, for which all participants will be invited to submit. The aim of this special issue will be to collect different perspectives on the identified challenges, trigger research in the field of EUD to pursue those, and raise awareness of the relevance and importance of EUD research.

\section{References}

[1] Atzori, L., Iera, A., and Morabito, G. The Internet of Things: A Survey. Comput. Netw. 54, 15 (2010), 27872805.

[2] Blackwell, A. and Burnett, M. Applying attention investment to end-user programming. Proc. $\mathrm{HCC} \mathrm{C}^{\prime} 02$ (2002), 28-30.

[3] Burnett, M.M. and Myers, B.A. Future of End-user Software Engineering: Beyond the Silos. Proceedings of the on Future of Software Engineering, ACM (2014), 201-211.

[4] Danado, J. and Paternò, F. Puzzle: A Mobile Application Development Environment Using a Jigsaw Metaphor. J. Vis. Lang. Comput. 25, 4 (2014), 297315.

[5] Fischer, G. End User Development and MetaDesign: Foundations for Cultures of Participation. J. Organ. End User Comp. 22, 1 (2010), 52-82.

[6] Lieberman, H., Paternò, F., Klann, M., and Wulf, V. End-User Development: An Emerging Paradigm. In $\mathrm{H}$. Lieberman, F. Paternò and V. Wulf, eds., End User Development. Springer Netherlands, Dordrecht, 2006, 1-8.

[7] Maceli, M. and Atwood, M.E. From Human Crafters to Human Factors to Human Actors and Back Again: Bridging the Design Time - Use Time Divide. In M.F. Costabile, Y. Dittrich, G. Fischer and A. Piccinno, eds., End-User Development. Springer Berlin Heidelberg, Berlin, Heidelberg, 2011, 76-91.

[8] De Souza, C.S., Garcia, A.C.B., Slaviero, C., Pinto, H., and Repenning, A. Semiotic traces of computational thinking acquisition. Proc. IS-EUD 2011, SpringerVerlag (2011), 155-170

[9] Tetteroo, D., Markopoulos, P., Timmermans, A. and Seelen, $\mathrm{H}$. Rehabilitation Therapists as Software Creators? Introducing End User Development in a Healthcare Setting. Int J of Sociotechnology and Knowledge Development 6, 1 (2014), 36-50. 